\title{
OPTIMIZATION OF RESOURCE ALLOCATION IN R+D PROJECTS
}

\author{
Ryszard Waśniowski \\ Technical University of Wrocław \\ Wrocław, Poland
}

While formulating $R+D$ programmes we came across the problem of optimal resource allocation among separate disciplines concerned with realization of a defined number of subjects and tasks. Optimal decisions on financing the research projects is a much complex problem and requires the application of mathematical programming algorithms. To use the algorithms of optimal resource allocation such input values as realization costs of a given subject, probability of its effective realization, and its priority within the whole research programme should be definied. The estimation accuracy of the input data depends on the assumed optimization time horizon. For this reason resource allocation methods should be classified according to the models used, i.e. either static or dynamic ones.

\section{Formulation of the problem}

We assume the programme of research aiming to achieve the realization of global goal to be defined. The programme is constituted by subjects subdivided into particular tasks. By an optimal resource allocation we mean the distribution of limited budget among the separate subjects that ensures the maximum quality index of global problem.

\section{Static models of resource allocation into $R+D$ projects}

Static models are used to establish the programmes of $R+D$ projects for time periods not longer than $3 \div 6$ years. This constraint results from the fact that at a longer optimization timehorizon the input data to a given model lose their validity becoming stale. Let us assume that the research programme consiwts of a set of $\mathrm{N}$ projects. For the above set we have defined the vector of weights $a=\left(a_{1}, a_{2}, \ldots, a_{N}\right)$, where $a_{i}$ denotes the weight of the $i-t h$ project from the set $N$. The realization cost of a separate project is given by the vector $k=\left(k_{1}, k_{2}, \ldots k_{N}\right)$, $k_{i}$ being the cost of the $i-t h$ project. Both weight and cost of separate projects are estimated by the experts. 
Let us introduce zero-one variable, then

$$
x_{i}= \begin{cases}1, & \text { if the } i-t h \text { project was accepted to realization, } \\ 0, & \text { otherwice }\end{cases}
$$

Optimization problem is reduced to determining the set $x=\left(x_{1}, x_{2}, \ldots, x_{N}\right)$ with respect of the maximum of quality criterion

$$
J=\max _{\left\{x_{i}\right\}} \sum_{x=1}^{N} a_{i} x_{i}
$$

at budget constraint $\sum_{i=1}^{N} k_{i} x_{i} \leqslant B$,

where B-budget assigned to the realization of the programme.

To solve this problem the Balas, Dragan, and Mylen [1] procedures have been used. The solution of linear zero-one programming problem allows to obtain collection selected from the set $\mathrm{N}$ projects differing in validity and realization cost.

In mathematical models of optimal resource allocation a specific character of the programme of research project should be often taken into consideration. Consequently, such models can be applied solely to a defined class of problems e.g. a static model for the optimization of allocation of resources designed for the realization of the collection of projects conditioning the achievement of defined military goals has been presented by Dean and Hauser [2]. By simulating the model on computer and by repeatedly solving the problem at different levels of global budget the global quality index can be determined as a function of buaget $J(B)$. This allows to establish the upper limit of the budget. Above this limit any financing of the research projects becames inexpedient.

\section{Resource allocation based on aggregation of the project networks}

At first let us introduce some basic definitions. The activity is a proces described by the following equation:

$$
v_{i}(t)=\frac{d x_{i}(t)}{d t}=f_{i}\left[h_{i j}(t), t\right]
$$

where: $x_{i}(t)$ is the state of the $i$-th activity at the moment $t ; x_{i}\left(t_{0}\right)=0$, $x_{i}\left(t_{k}\right)=w_{i}, t_{0}$ and $t_{k}$ are the initial and the final moments of an activity, respectivelly, $w_{i}$ is the volume of the $i$-th activity, $v_{i}(t)$ is 
the rate of the $i-t h$ activity at the moment $t, h_{j j}(t)$ are the resources group parameters of the $i$-th activity, $h_{i}(t)=\left\{h_{i j}(t)\right\}$ is the m-vector of the i-th activity resource group. If the modulus of the vector $h_{i}(t)$ depends only on the value $t$, then the latter can be represented as $h_{i}(t)=p_{i}(t) a_{i}$, where $p_{i}(t)$ is the power of the $i$-th activity resource group at the moment $t, \alpha_{i}=\left\{\alpha_{i j}\right\}$ is the m-vector of resource group parameters for the $i-t h$ activity, and resource group paraneter $\alpha_{i j}$ is the value of the $j$-th resource when $\rho_{i}(t)=1$. Project is a set partially ordered consisting of final number of activities. It can be represented by a network. The project is said to be realized if its state $x(t)$ changes its value from the initial value $x(0)=0$ up to the final value $x(T)=W$ being the volume of the project, and $T$ the final moment of the project. We will assume further that the project is finished if all its activities are finished. Multiproject is a set of independet projects, which must be realized by common resources. There are two types of resource constraints.

$$
\begin{aligned}
& \sum_{p=1}^{1} \sum_{i=1}^{n_{p}} \alpha_{i j} \rho_{i}(t) \leqslant N_{j}(t), j=1,2, \ldots, m, \\
& \sum_{p=1}^{1} \sum_{i=1}^{n}{ }^{\alpha_{i j}} q_{i} \leqslant s_{j}, j=1,2, \ldots, m .
\end{aligned}
$$

where $q_{i}=\int_{0}^{T} \rho_{i}(t) d t$ is the power consumption of the $i$-th activity $\left(i=1,2, \ldots, n_{p}\right), s_{j}$ is the permissible consumption of the $j-t h$ resource in the multiproject, $\mathrm{N}_{j}$ is the given value of the $j-t h$ resource in the multiproject at the moment $t, i$ is the number of the project activity $\left(i=1,2, \ldots, n_{p}\right), p$ is the number of the project $(p=1,2, \ldots, 1)$. The project aggregation is a network of projects represented by one activity.

Let us assume that the multiproject consisting of 1 projects, each having the volume $\mathrm{w}_{1}, \mathrm{w}_{2}, \ldots, \mathrm{w}_{1}$, must be realized under definite constraints of resources. The problem is to allocate the resources to project activities so that the criterion (1) or (2) be minimized. As criterion several functions may be used e.g. $\min \max \mathrm{T}_{\mathrm{p}}$ and $\sum_{p=1}^{1} \beta_{p}\left(T_{p}\right)$, where $T_{p}$ is the final moment of the $p$-th project and $\beta_{p}\left(T_{p}\right)$ is a non-decreasing function of the $T_{p}$. The aggregation of the project networks permits to obtain the solution of the problem as a sequence 
of the following actions: I Aggregate the project networks, i.e. given the values of $w_{i}, \alpha_{i}$ and functions $v_{i}(t)=f_{i}\left[p_{i}(t), t\right]$ for each activity, define the values of $w_{p} \alpha_{p}$ and functions $v_{p}(t)=f_{p}\left[\rho_{p}(t), t\right]$. II when aggregation is completed solve the resource allocation problem with 1 independet activities. This step gives the values of $\mathrm{N}_{\mathrm{pj}}(\mathrm{t})$ and $S_{p j}$ for each project.

III Using the values found in previous step solve the allocation problem for each project separately.
Thus the problem of resource allocation with $n=\sum_{p=1}^{1} n$ activities is transformed to 1 resource allocation problems with $n_{p}$ activities. As an example of such an approach consider the solution of the resource allocation problem in the multiproject, when the resources are of financial means and the criterion min $\max _{\mathrm{p}} \mathrm{T}_{\mathrm{p}}$. It is assumed that each activity in the network is subject to a continuous upward-concave time-cost relationship. Each $S_{p}\left(T_{p}\right)$ being a non decreasing function of $T_{p}$, all the projects have the some final moment, i.e. $T_{1}=T_{2}=\ldots=T_{1}=T_{\text {, }}$ if the final moment of the multiproject is minimized. Hence, the value of $T$ may be found from the equation $S_{1}(T)=$ $=S_{2}(T)=\ldots S_{1}(T)=S$.

\section{Dynamic models of optimal resource allocation into $R+D$ projects}

The problem of optimal financial allocation of resources among separate realization periods of one project can be also solved by a dynamic programing method [3].

Let us assume that the project (programme) is to be realized in the course of $\mathrm{N}$ periods, e.g. during $\mathrm{N}$ years.

Let us introduce the following denotations:

$x_{i}$-budget assigned for the i-th (from the end) period of time, $y_{i}$-budget used till the $i$-th period of time, i.e. in the course of $N-i$ time intervals,

$Q_{i}$-expected value of the income at the accomplishment of the project in the i-th (from the end) period of time, i.e. the difference between the expected market and current prices, $P\left(x_{i}, y_{i}\right)$ - probability of realization of projects in the $i-t h$ (from the end) period of time as a function of $x_{i}, y_{i}$,

$c_{i}$ - market price of the project, provided that the latter was realized in the $i-t h$ (from the end) period of time.

The problem of optimalization consists here in an optimal budget allocation to the separate periods of project realization, i.e. in deter- 
mining an optimal vector $x_{1}^{*}, x_{2}^{*}, \ldots, x_{r}^{*}$ with respect to the expected value of the income consummed. Given initial budget $x_{N}$ the optimal sequence $\mathrm{x}_{\mathrm{N}-1}^{*}, \mathrm{x}_{\mathrm{N}-2}^{*}, \ldots, \mathrm{x}_{1}^{*}$ will be obtained according to the Bellman recurrent algorithm:

$Q_{i}\left(y_{i}\right)=\max _{0 \leqslant X_{i}}\left\{C_{i} P_{i}\left(x_{i}, y_{i}\right)-x_{i}+r_{i}\left[1-P_{i}\left(x_{i}, y_{i}\right)\right] f_{i-1}\left(y_{i-1}\right)\right\}$

where $y_{i-1}=y_{i}+x_{i}$ and $\gamma_{i} \in[0,1]$ is coefficient of punishment for the delay in realization of project, fixed by the experts. Coefficient $\gamma_{i}$ can either be constant or increase with time. The component $\left[C_{i} P_{i}\left(x_{i}, y_{i}\right)-x_{i}\right]$ in (3) denotes the expected value of income, if the given project is in the $i$-th (from the end) period of time, whereas $\gamma_{i}\left[1-P_{i}\left(x_{i}, y_{i}\right)\right] f_{i-1}\left(y_{i-1}\right)$ is the expected value of the income in case when the project is finished in time interval following the $i-t h$ one from the end. Parameters $C_{i}$ and relations $P_{i}\left(x_{i}, y_{i}\right)$ are estimated by the experts.

\section{Conclusions}

The optimization methods of financing the research projects presented in the paper have been adopted by the Technical University of Wrocław. ALGOL programmes implementing the procedures described have been developed. A listing and implementation on the use of the programmes are available from the author. It should be emphasized that the application of static models is not related with any computational difficulties, while the methods of complex of operations and dynamic programming required complex numerical calculations. The performed investigations allow to state that more efficient algorithms of a dynamic resource allocation into $R+D$ should be found.

\section{References:}

[1] KUCHARCZYK J., SYSEO M., Algorytmy optymalizacji w jązyku ALGOL 60, (Algorithms of optimization in ALGOL 60), PWN Warszawa (in Polish).

[2] DEAN B.V., HAUSER L.E., Advanced Material Systems Planning. IEEE Trans.on Eng.Man. Vol.EM-14, NO 1, March 1967.

[3] BELLMAN R.E., DREYFUS S.E., Applied Dynamic Programming. Princeton University Press, Princeton, New Jersey, 1963. 